\title{
A thermochromic tissue-mimicking phantom model for verification of ablation plans in thermal ablation
}

\author{
Yan Zhou ${ }^{1,2,3 \#}$, Lin Zhao ${ }^{2,3 \#}$, Xinyu Zhong ${ }^{4}$, Jianmin Ding ${ }^{2,3}$, Hongyu Zhou ${ }^{2,3}$, Fengmei Wang ${ }^{3,5}$, \\ Xiang Jing ${ }^{2,3}$ \\ ${ }^{1}$ School of Medicine, Nankai University, Tianjin, China; ${ }^{2}$ Department of Ultrasound, Tianjin Third Central Hospital, Tianjin, China; ${ }^{3}$ Tianjin \\ Institute of Hepatobiliary Disease, Tianjin Key Laboratory of Extracorporeal Life Support for Critical Diseases, Artificial Cell Engineering \\ Technology Research Center, Tianjin Third Central Hospital, Tianjin, China; ${ }^{4}$ The Third Affiliated Hospital of Tianjin Medical University, Tianjin, \\ China; ${ }^{5}$ Department of Gastroenterology and Hepatology, Tianjin Third Central Hospital, Tianjin, China \\ Contributions: (I) Conception and design: X Jing, Y Zhou, L Zhao; (II) Administrative support: X Jing; (III) Provision of study materials or patients: \\ Y Zhou, L Zhao, X Zhong; (IV) Collection and assembly of data: Y Zhou, L Zhao, X Zhong; (V) Data analysis and interpretation: Y Zhou, L Zhao; \\ (VI) Manuscript writing: All authors; (VII) Final approval of manuscript: All authors. \\ \#These authors contributed equally to this work. \\ Correspondence to: Xiang Jing. Tianjin Third Central Hospital, Hedong District, No. 83 Jintang Road, Tianjin 300170, China. \\ Email: dr.jingxiang@aliyun.com.
}

Background: Our study aims to develop a novel tissue-mimicking thermochromic with tumor model for visualization of thermal ablation and verification of ablation plans

Methods: Polyacrylamide gel was mixed with thermochromic ink to produce a phantom model. A phantom model embedded in a tumor model was constructed and used to evaluate the ablation procedure. The phantom models were randomly divided into complete ablation group and incomplete ablation group. The ablation planning of the tumor was on the 3D US and performed on a phantom model. We guide the ablation procedures according to the ablation planning. The results measured in a gross specimen of the phantom model were compared with the expected results in ablation planning.

Results: The color of the model changes from cream white to magenta after heating. The mono-site ablation area is a spheroid after thermal ablation with a size of $3.0 \times 1.8 \mathrm{~cm}$ at $60 \mathrm{~W}, 5$ minutes, $3.5 \times 2.5 \mathrm{~cm}$ at $60 \mathrm{~W}, 10$ minutes, and $4.0 \times 3.5 \mathrm{~cm}$ at $60 \mathrm{~W}, 15$ minutes, respectively. According to the ablation planning, a total of 4 ablation points were needed to retrieve the complete ablation of a $3.0 \mathrm{~cm}$ tumor. The complete ablation and incomplete ablation were proved by a gross specimen of the phantom model as we expected

Conclusions: A novel thermochromic tissue-mimicking phantom model with a spherical tumor model has been designed and developed. The ablation area can be visualized on this phantom model by the permanent color change. This phantom model can assess the ablation planning system's accuracy and train operators for ultrasound-guided thermal ablation.

Keywords: Phantom model; thermal ablation; ablation planning; ultrasound

Submitted Jan 19, 2021. Accepted for publication Feb 20, 2021.

doi: $10.21037 / \mathrm{atm}-21-523$

View this article at: http://dx.doi.org/10.21037/atm-21-523

\section{Introduction}

Thermal ablation, including radiofrequency ablation (RFA), microwave ablation (MWA) and laser ablation, was widely used for early hepatocellular carcinoma (HCC) $(1,2)$.
The principle of thermal ablation is that thermal energy produced by RFA or MWA causes coagulative necrosis of HCC to achieve the purpose of radical treatment (3). The necrosis area must cover the tumor completely during an 
ablation procedure. Once a residual tumor exists, it will cause the growth and invasion of HCC and hinder patients from a satisfactory prognosis (4).

It is easy for a small HCC to retrieve complete ablation because only one or a few ablation sites are needed to cover the whole tumor (5). However, for the large tumors, especially for the tumor with a size larger than $5 \mathrm{~cm}$, the incomplete ablation rates increase markedly $(6,7)$. With the increase of tumor size, the ablation volume and ablation points needed to retrieve complete ablation grow exponentially. Multiple overlap ablation with a mathematical ablation model was presented in clinical practice to improve the treatment outcome of thermal ablation for large HCC $(8,9)$. However, this ablation protocol is, to some extent, experience-dependent. It is difficult for most beginners of image-guided thermal ablation, who may spend a long time passing the learning curve. We always hope the experience of thermal ablation was acquired from something else instead of patients.

The other advanced method to assist thermal ablation for the large tumor is ablation planning, in which the ablation points and puncture path can be programmed automatically with the preoperative $3 \mathrm{D}$ image of the tumor. Several commercial ablation planning systems are achievable in our daily work, including a 3D visualization ablation planning system, a 3D ultrasound image ablation planning system, and a CT based ablation planning system (10-13). More developing ablation systems are on the way. However, these ablation planning systems' accuracies can be difficult to evaluate since there is insufficient assessment.

A tissue-mimicking phantom model can address the issues mentioned above and plays a significant role in ablation procedures (14-16). The optimal tissue-mimicking phantom model may have the following characteristics. First, it is with the chemical and physical long-term stability. Second, some physical properties, including specific heat capacity and susceptibility to puncture, like those of human tissues. Third, accurate visualization of the coagulation lesion after thermal ablation. Fourth, a repeatable ablation size with the same ablation power and time. Fifth, a tumor model embedded in the parenchyma model can be distinguished by the US before ablation and distinguished on the gross specimen after thermal ablation. Hence, we aim to develop a tissue-mimicking phantom model with the characteristics above.

This study developed a thermochromic tissue-mimicking phantom model through a polyacrylamide gel and commercially available thermochromic ink, which can assess the ablation planning system's accuracy by displaying the ablation region and training the operators for ultrasoundguided thermal ablation. We present the following article in accordance with the MDAR reporting checklist (available at http://dx.doi.org/10.21037/atm-21-523).

\section{Methods}

\section{Phantom fabrication}

This phantom is formed of a polyacrylamide gel mixed with commercially available thermochromic ink $(17,18)$. This ink permanently changes color from cream white to magenta as temperature increases. According to the ink manufacturer, the maximum color density is reached at around $60^{\circ} \mathrm{C}(19)$. A cross-linking copolymerization makes the polyacrylamide gels of acrylamide and N, N'-methylene bisacrylamide (bis) in an aqueous solution.

The following protocol for phantom fabrication was used: (I) the acrylamide and N, N'-methylene bis with a 19:1 ratio added ultrapure water to get a mixture consisting of a $7 \%$ acrylamide:bis concentration. (II) These steps were then followed by the addition of thermochromic ink with $5 \%(\mathrm{v} / \mathrm{v})$. (III) ammonium persulfate was added to initiate polymerization with $0.14 \%$ (w/v). (IV) $\mathrm{N}, \mathrm{N}, \mathrm{N} 0, \mathrm{~N} 0$ tetramethylethylenediamine was added with $0.1 \%(\mathrm{v} / \mathrm{v})$. The final solution was immediately transferred to a plastic container of the desired size and kept in a cold room $\left(4^{\circ} \mathrm{C}\right)$ overnight.

Two Phantom models were established according to our aims. One phantom model was without a spherical tumor model, and another phantom model included a spherical tumor model with a size of $3.0 \mathrm{~cm}$. The spherical tumor model can be distinguished from the background model clearly by the US.

\section{Model testing}

The phantom was used to assess the MWA procedure. Three aims of this testing were included. First, to test the color change of phantom with the high temperature caused by MWA. Second, the phantoms were ablated by MWA at different power and time to assess if the ablation size was ablation power and time-dependent. Third, a repeat ablation procedure is performed to determine if a stabilized ablation size can be retrieved at the same power and time. If it can be retrieved, the ablation size is recorded for the next step. 


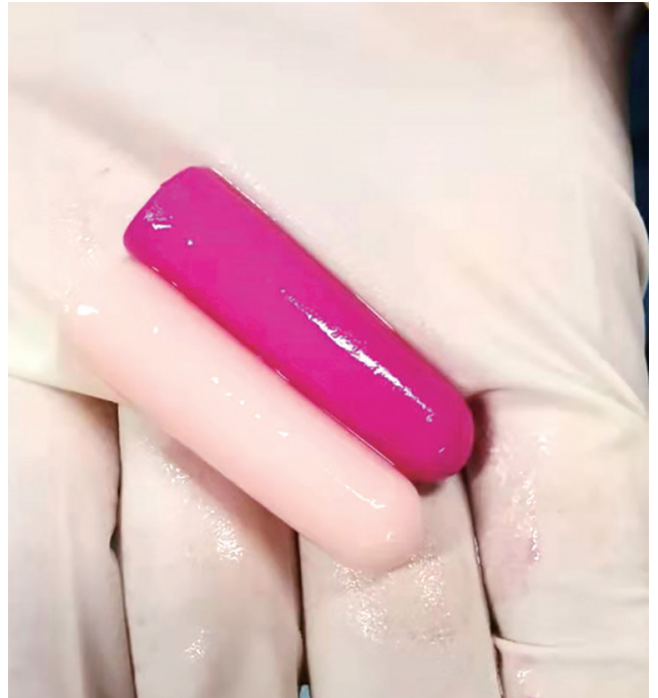

Figure 1 The color differences of phantom samples with (magenta one) and without (cream white one) hot water bath.

\section{Study grouping}

The phantom model included a spherical tumor model with a $3.0 \mathrm{~cm}$ size to test complete or incomplete ablation procedure. The phantom models were randomly divided into two groups, including a complete ablation group in which the tumor model covered the ablative area and an incomplete ablation group in which the tumor model did not cover the ablative area.

\section{Ablation planning}

Before the ablation, 3D ultrasound volumes were acquired and reconstructed using the PercuNav feature of the Philips EPIQ 7 ultrasound system. First, three-dimensional ultrasound image data for the spherical tumor model were collected. Second, the spherical tumor model and background model were segmented. After that, the 3-D ultrasound images in an arbitrary scan view or a rendered volume to segment the spherical tumor model and background model can be reviewed. The computer-assisted tumor ablation planning for 3-D ultrasound was then performed before the ablation to complete the ablation. The mono-site ablation size used for ablation planning was on the results of the last step. JM performed the ablation planning procedure. $\mathrm{D}$ who had $10 \mathrm{y}$ of experience with ultrasound-guided ablation for HCC.

\section{MWA procedures}

MWA procedures were carried out using an MTC3 microwave therapy instrument (Forsea Microwave \& Electronic Research Institute, Nanjing, China) with a frequency of 2,450 MHz and an output power of 50-100 W. The microwave (MW) antenna was a $14 \mathrm{G}$ unipolar cooledshaft needle $15 \mathrm{~cm}$ with a $1.5 \mathrm{~cm}$ active tip.

MWA was carried out under ultrasound guidance. MWA output power was $60 \mathrm{~W}, 10$ minutes per point. Multiple points ablation was performed according to preoperative planning. For research purposes, a complete ablation was performed exactly as planned. An incomplete ablation procedure was performed as planned, but one ablation site was retained to evaluate the residual spherical tumor model. All the ablation procedures were performed respectively by a senior operator (JM.D) who had $10 \mathrm{y}$ of experience with ultrasound-guided ablation for HCC.

\section{Evaluation of ablation margin}

The phantom models were sliced imminently after thermal ablation to assess the ablation region. The result of the phantom model was compared with the ablation planning we expected.

\section{Statistical analysis}

Data were expressed as median \pm standard deviation (SD) (range). All data were analyzed using SPSS version 22.0 software (SPSS, Chicago, IL, USA).

\section{Results}

Two conical phantom samples were prepared using the above process. One of these phantom samples was then equilibrated to ambient temperature and heated at $65^{\circ} \mathrm{C}$ for 10 minutes using a hot water bath. Another phantom sample without a hot water bath was kept at room temperature. Photographs of both the heated and unheated samples were taken to illustrate the color differences. We can observe the color of the model changes from cream white to magenta (Figure 1).

The cuboid parenchyma models with a size of $(6 \times 8 \times 8)$ $\mathrm{cm}$ were abated by MWA at $60 \mathrm{~W}$ with different times, including 5,10,15 minutes. The ablation area is a spheroid, like the HCC ablated zone. The mono-point ablation size 
Table 1 Mono-site ablation size at $60 \mathrm{~W}$ with different time

\begin{tabular}{lccc}
\hline Size & $\begin{array}{c}60 \mathrm{~W}, \\
5 \text { minutes }\end{array}$ & $\begin{array}{c}60 \mathrm{~W}, \\
10 \text { minutes }\end{array}$ & $\begin{array}{c}60 \mathrm{~W}, \\
15 \text { minutes }\end{array}$ \\
\hline Long radius $(\mathrm{cm})$ & 3.0 & 3.5 & 4.0 \\
Short radius $(\mathrm{cm})$ & 1.8 & 2.5 & 3.5 \\
\hline
\end{tabular}

was time-dependent at the same output power (Table 1, Figure 2A).

The ablation procedures were performed six times repeatedly at $60 \mathrm{~W}$ at different times (Table 2, Figure 2B). The mono-site ablation area is a spheroid after thermal ablation with a size of $3.0 \times 1.8 \mathrm{~cm}$ at $60 \mathrm{~W}, 5$ minutes, $3.5 \times 2.5 \mathrm{~cm}$ at $60 \mathrm{~W}, 10$ minutes, and $4.0 \times 3.5 \mathrm{~cm}$ at $60 \mathrm{~W}$, 15 minutes, respectively.

When a $3.0 \mathrm{~cm}$ spherical tumor model is embedded in a cuboid parenchyma model, the US can detect the tumor model because the air gap between the tumor model and parenchymal model was hyper-echoic. Thus, 3D ultrasound volumes were acquired and reconstructed using the PercuNav system (Figure 3). Then ablation planning was performed. The mono-point ablation size used in the ablation planning system was $3.5 \times 2.5 \mathrm{~cm}(60 \mathrm{~W}$, 10 minutes). Four ablation sites were needed to achieve the complete ablation according to the ablation planning (Figure 4). According to our purpose, two ablation groups, the complete ablation group, and the incomplete ablation group, were developed. For the complete ablation group, the ablation producer was performed exactly as planned to achieve complete ablation. The sliced tumor and parenchyma model show that the ablation area (magenta) covered the tumor model completely, as we expected (Figure $5 A, B$ ). Only 3 ablation points were performed for the incomplete ablation group as planned during the procedure to achieve incomplete ablation. The sliced tumor and parenchyma model show that the ablation area (magenta) did not cover the tumor model completely, as we expected (Figure 5C,D).

\section{Discussion}

In this study, we developed a thermochromic tissuemimicking phantom model to assess the ablation region after MWA. This phantom model was on a polyacrylamide gel and commercially available thermochromic ink, which endowed our models with several major characteristics for simulated liver tissue properties during an ablation procedure.

Our phantom model contains primarily polyacrylamide gel, which has physical properties like liver tissues (18). First, the mass density and flexibility of polyacrylamide gel is like soft tissues. When a phantom model is punctured, it has the real feeling of puncturing liver tissue. The phantom model is hypo-echoic on the US, and a puncture needle or ablation electrode can be displayed under ultrasound guidance. Thus, it contributes significantly to the accumulating of study experience for ultrasoundguided interventional puncture. Second, the specific heat capacity, thermal conductivity, and electrical conductivity of polyacrylamide gel are comparable to biological tissue, as proved by earlier studies $(18,20)$, representing the phantom model as an ideal replacement of liver tissue for thermal ablation experiment investigations.

When the polyacrylamide gel model is used for thermal ablation, a critical issue that needs to be solved is how to visualize the ablation region of thermal ablation. Thermochromic materials may be used as indicators to address the problem $(21,22)$. In this study, commercially available thermochromic ink, the other composition of the phantom model, was used. The most important thermochromic ink character is thermochromism, a phenomenon in which a substance changes its color because of a temperature change. According to the ink manufacturer, color changes from cream white to magenta when temperatures above $60{ }^{\circ} \mathrm{C}$, reaching the maximum color density at around $60-65^{\circ} \mathrm{C}(19)$. This phenomenon can describe the process of coagulative necrosis of tumor or liver tissue ablated by thermal ablation.

The color change for thermochromic ink used in our study is permanent. Even upon cooling, the color is irreversible, so we can analyze our results. The color difference of the background region and ablation region will not disappear.

The polyacrylamide gel mixed with thermochromic ink creates an appropriate phantom model used for thermal ablation investigation. As our results show, because of thermochromic ink's color change, the ablation region can be displayed clearly in the phantom model. The ablation shape of MWA in the phantom model is a spheroid like that in liver tissue. With an increase of ablation time, the ablation area enlarged, reflecting the superior thermal conductivity and electrical conductivity of the polyacrylamide gel model and can be used to visualize the three-dimensional temperature distribution of thermal ablation. All these properties mentioned above make the 
A
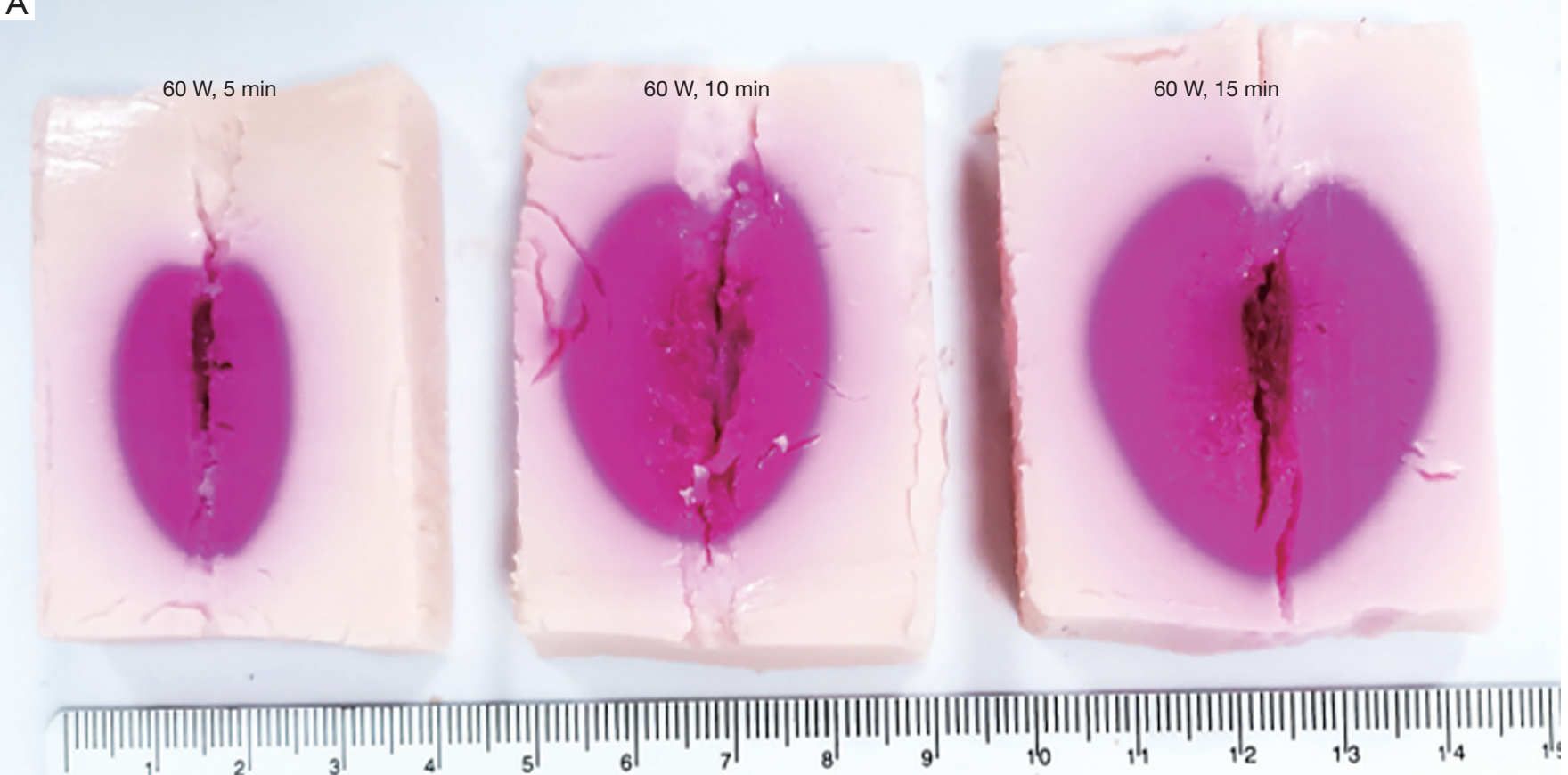
CENTIMETERS

\section{F R E N C H}

C A T HET ER

S C A L E

B

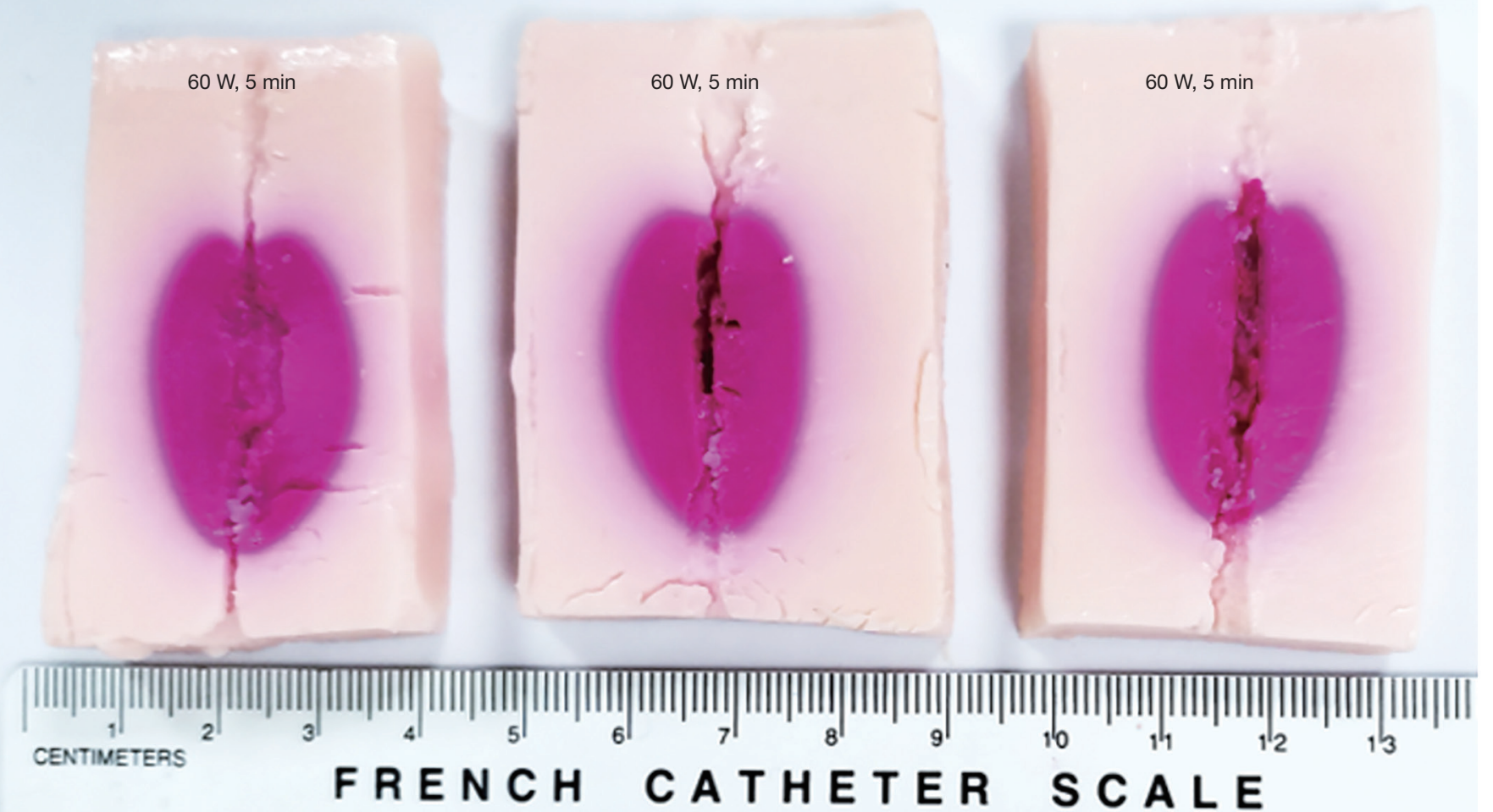

Figure 2 The gross specimen of the phantom model after MWA. (A) Phantom samples were ablated with $60 \mathrm{~W}$ at a different time; (B) phantom samples were ablated with the same power and time at $60 \mathrm{~W}, 5$ minutes. 
Table 2 Mono-site ablation size at $60 \mathrm{~W}$ with different time

\begin{tabular}{|c|c|c|c|c|c|c|c|}
\hline Size & $1^{\text {st }}$ & $2^{\text {nd }}$ & $3^{\text {rd }}$ & $4^{\text {th }}$ & $5^{\text {th }}$ & $6^{\text {th }}$ & Mean value \\
\hline \multicolumn{8}{|l|}{$60 \mathrm{~W}, 5$ minutes } \\
\hline Long radius $(\mathrm{cm})$ & 3.0 & 3.0 & 2.9 & 3.0 & 2.9 & 3.0 & $3.0 \pm 0.05$ \\
\hline Short radius $(\mathrm{cm})$ & 1.8 & 1.9 & 1.8 & 1.9 & 1.8 & 1.8 & $1.8 \pm 0.05$ \\
\hline \multicolumn{8}{|l|}{$60 \mathrm{~W}, 10$ minutes } \\
\hline Short radius (cm) & 2.5 & 2.5 & 2.6 & 2.5 & 2.5 & 2.4 & $2.5 \pm 0.06$ \\
\hline \multicolumn{8}{|l|}{$60 \mathrm{~W}, 15$ minutes } \\
\hline Long radius (cm) & 4.0 & 3.9 & 4.0 & 4.0 & 3.9 & 3.9 & $4.0 \pm 0.05$ \\
\hline Short radius $(\mathrm{cm})$ & 3.4 & 3.4 & 3.5 & 3.4 & 3.5 & 3.6 & $3.5 \pm 0.06$ \\
\hline
\end{tabular}

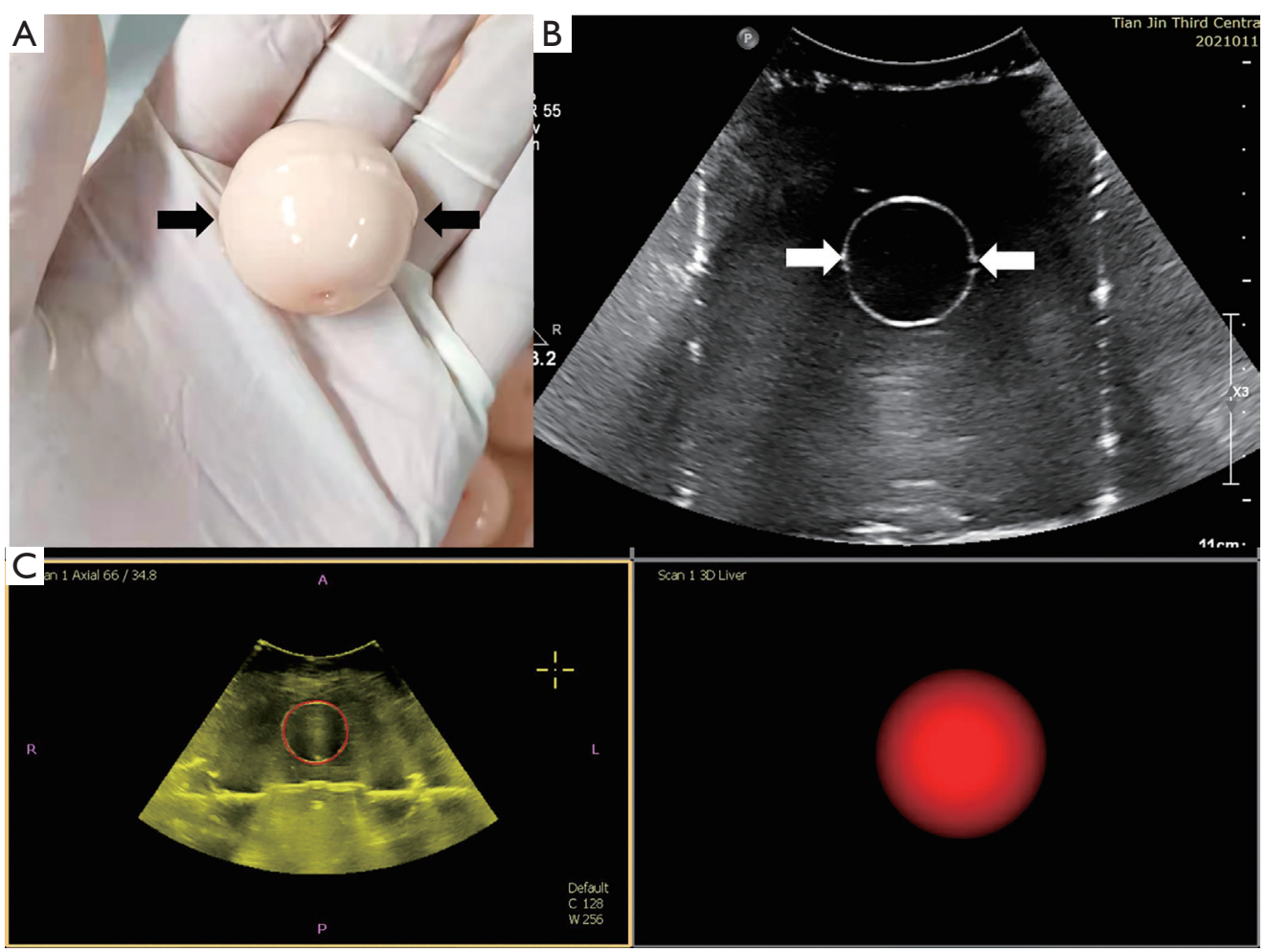

Figure 3 The US and 3D US images of the spherical tumor model. (A) Photo of a $3.0 \mathrm{~cm}$ spherical tumor model ( $\uparrow$ ); (B) the US illustrates the tumor model $(\uparrow)$; (C) 3D ultrasound image of the tumor model embedded in the parenchyma model.

phantom model an ideal training tool for thermal ablation.

In this study, we "planted a tumor" in our parenchymal model successfully, like an HCC lesion located in the liver. Although the tumor model and background model both are hypo-echoic in the US, the spherical tumor model can be distinguished from the background model because of the hyper-echoic of the tumor model margin. The air gap causes this hyper-echoic margin between the tumor model and the background model. Thus, the tumor model and background model can be segmented, and the tumor model's 3D volume data can be detected. Then ablation planning and ultrasound-guided thermal ablation can be 


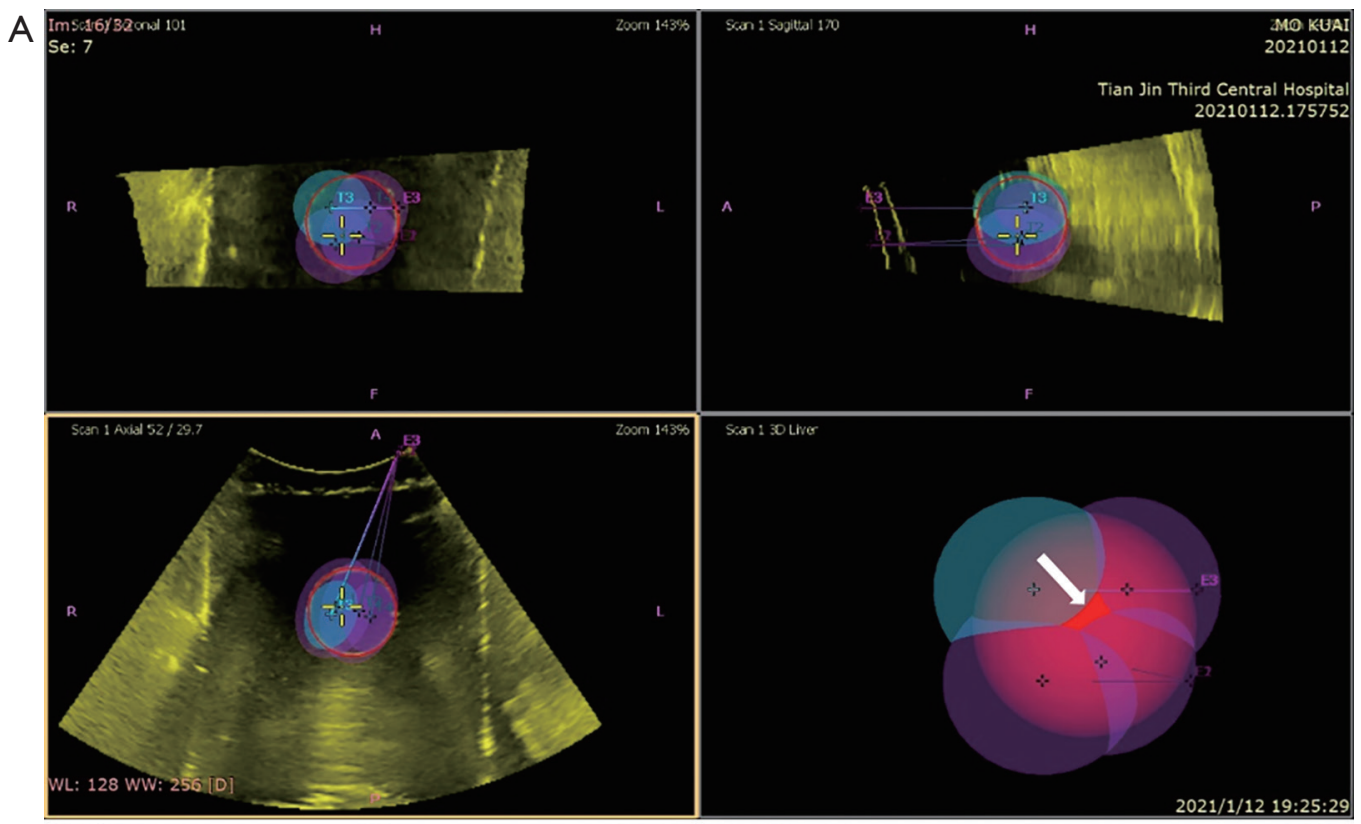

B
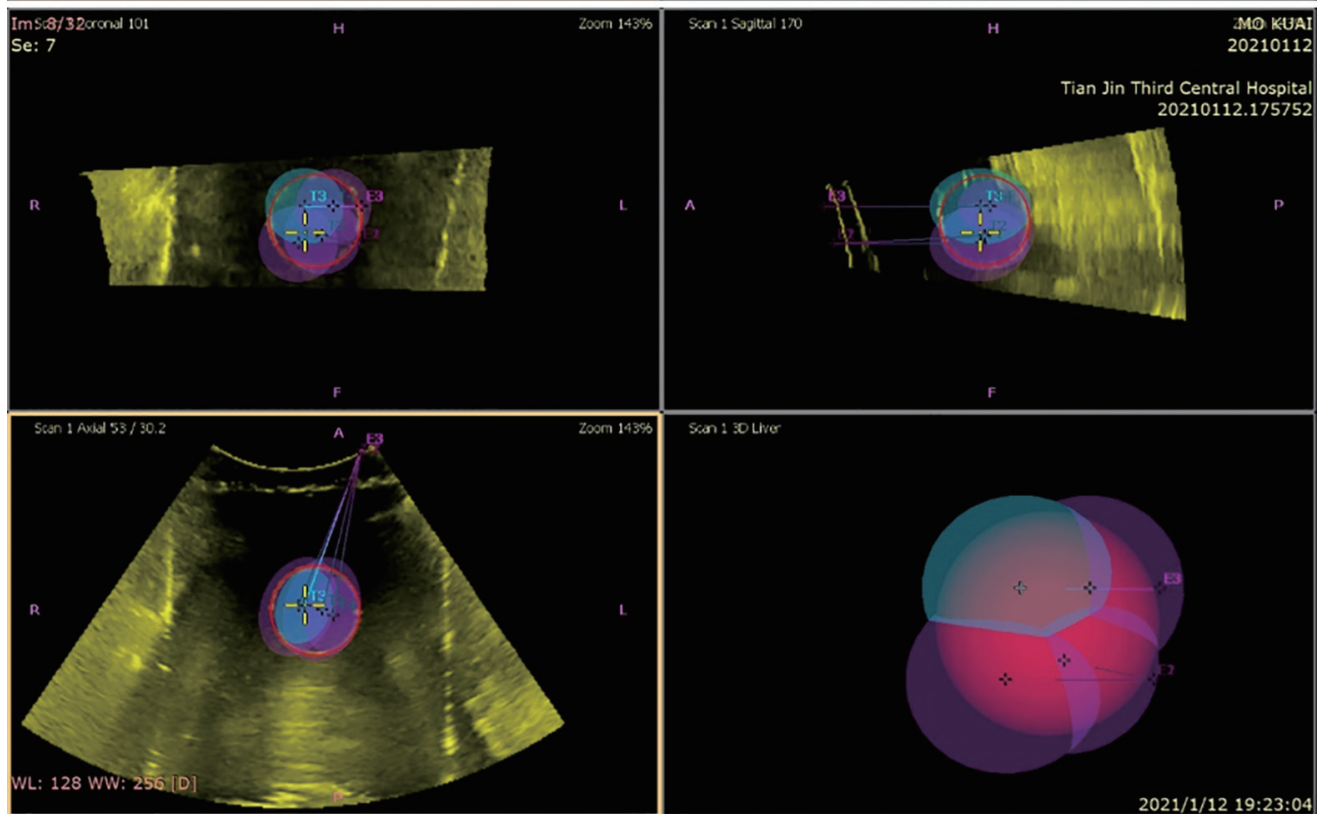

Figure 4 Ablation planning of the spherical tumor model. (A) An incomplete ablation ( $\uparrow$ ) can be displayed on 3-D ultrasound images during the ablation planning procedure; (B) a complete ablation can be achieved with 4 points ablation.

performed on a thermochromic tissue-mimicking phantom model. The other favorable feature of this phantom model is that different colors can distinguish the ablation region. The tumor model can also be separate from the background model on the gross specimen after thermal ablation because of the shrinking, enabling the phantom model to evaluate whether the tumor has been completely ablated.
We can achieve several goals through these thermochromic tissue-mimicking phantom models. First, it can be used to test the therapeutic characteristics of ablation devices. Second, accuracy verification of ablation plans can be achieved by evaluating the ablation region after RFA or MWA. Third, it is a teaching appliance in the thermal therapy study and can be used for personnel training. 


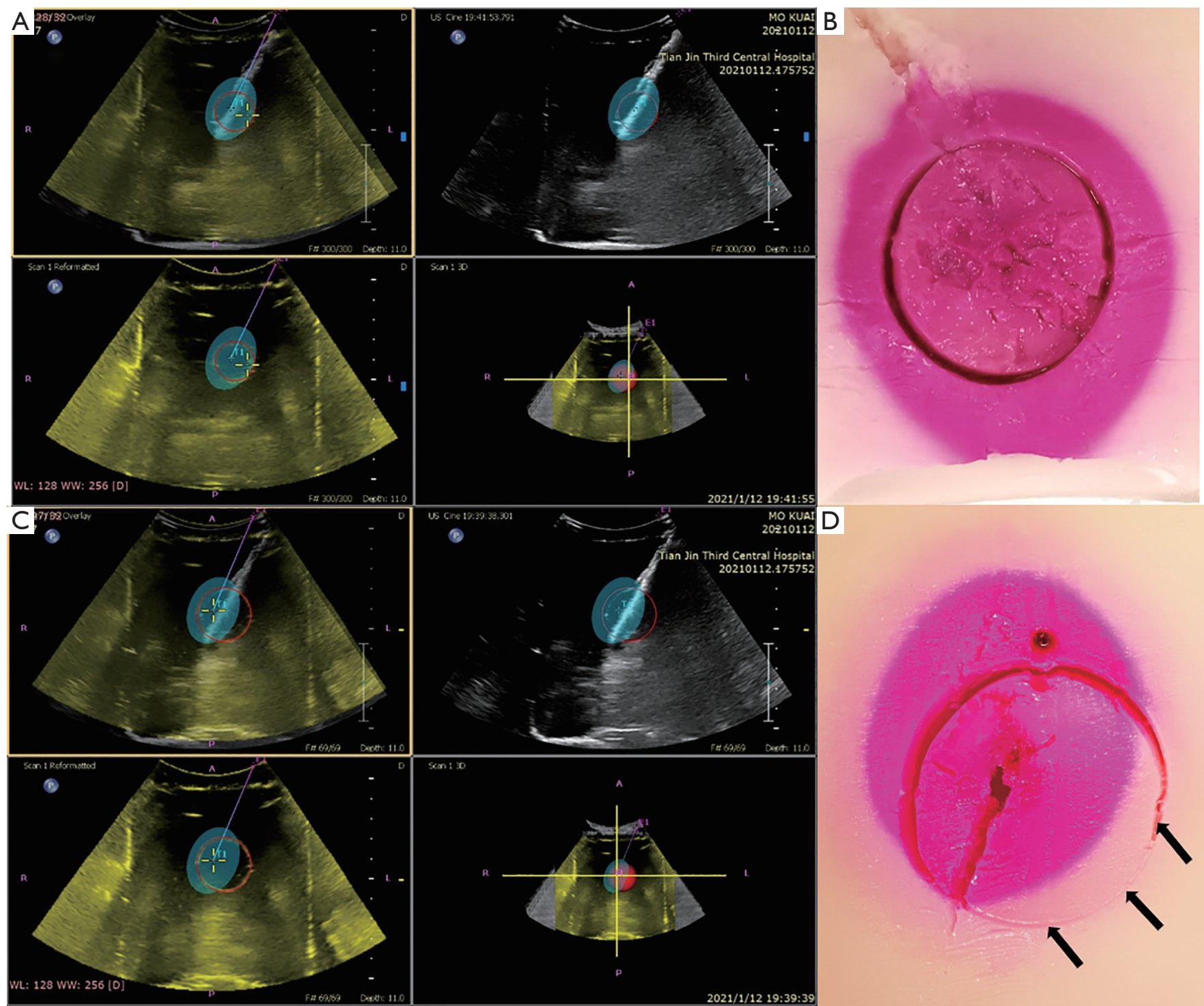

Figure 5 The expected results in ablation planning with the corresponding gross specimen of the phantom model. (A) Ablation producer was performed exactly as planned to retrieve complete ablation; (B) the complete ablation was verified on the phantom model; (C) only 3 ablation points were performed as planned to retrieve incomplete ablation; (D) the incomplete ablation was verified, and the residual tumor model $(\uparrow)$ was displayed on a phantom model.

Other phantom models were also developed in previous studies. Several studies have designed a phantom on a polyacrylamide gel mixed with BSA or egg white, a protein used as a temperature-sensitive indicator $(17,20,23)$. Although the ablation area can be displayed on the phantom model, the ablation area's margin is obscure because the coagulated gel turns into ivory white after ablation, without a significant color difference between the colorless background. The protocol for phantom fabrication used in the study of Negussie et al. (18) is like our study. Several ablation devices, including laser ablation and MWA, have been tested, and thermal properties and color change have been clarified in their study. However, the evaluation of ablation plans cannot be achieved because no tumor model is embedded in the parenchyma model. Li et al. (11) constructed a phantom model with carrageenan and an oral ultrasonic contrast agent to evaluate the ablation margin through a US-CT/MRI fusion image. Although, a spherical 
tumor model and an ablation margin model were embedded in the parenchyma model. After ablation, the liver tumor model and the ablation margin model, and a portion of the parenchyma model were melted and then replaced by the red liquid of the ablation zone solidified for evaluation. The process is complex, and only mono-point ablation instead of multiple overlap ablation was investigated.

There are still some limitations in our study and phantom model. First, the model can be used to evaluate complete or incomplete ablation but not the safety margin. Second, although the tumor model and parenchyma model can be distinguished in the US, they show the same hypoechoic in the US. Third, the ablation area cannot be detected by the US.

\section{Conclusions}

A novel thermochromic tissue-mimicking phantom model with a spherical tumor model has been designed and developed in this study. The ablation area can be visualized on this phantom model by the permanent color change of the model when the temperature above $60^{\circ} \mathrm{C}$. Thus, it can be used to assess the ablation planning system's accuracy and train operators in ultrasound-guided thermal ablation.

\section{Acknowledgments}

Funding: This work was supported by Tianjin health and Health Committee (Grant No. ZD20014, MS20017, KJ20170, NQ20033) and Tianjin Science and Technology Project (Grant No. 17ZXMFSY00170).

\section{Footnote}

Reporting Checklist: The authors have completed the MDAR reporting checklist. Available at http://dx.doi.org/10.21037/ atm-21-523

Data Sharing Statement: Available at http://dx.doi. org/10.21037/atm-21-523

Conflicts of Interest: All authors have completed the ICMJE uniform disclosure form (available at http://dx.doi. org/10.21037/atm-21-523). The authors have no conflicts of interest to declare.

Ethical Statement: The authors are accountable for all aspects of the work in ensuring that questions related to the accuracy or integrity of any part of the work are appropriately investigated and resolved.

Open Access Statement: This is an Open Access article distributed in accordance with the Creative Commons Attribution-NonCommercial-NoDerivs 4.0 International License (CC BY-NC-ND 4.0), which permits the noncommercial replication and distribution of the article with the strict proviso that no changes or edits are made and the original work is properly cited (including links to both the formal publication through the relevant DOI and the license). See: https://creativecommons.org/licenses/by-nc-nd/4.0/.

\section{References}

1. Forner A, Reig M, Bruix J. Hepatocellular carcinoma. Lancet 2018;391:1301-14.

2. Benson AB, D'Angelica MI, Abbott DE, et al. Guidelines Insights: Hepatobiliary Cancers, Version 2.2019. J Natl Compr Canc Netw 2019;17:302-10.

3. Goldberg SN, Gazelle GS, Mueller PR. Thermal ablation therapy for focal malignancy: a unified approach to underlying principles, techniques, and diagnostic imaging guidance. AJR Am J Roentgenol 2000;174:323-31.

4. Yin XY, Xie XY, Lu MD, et al. Percutaneous thermal ablation of medium and large hepatocellular carcinoma: long-term outcome and prognostic factors. Cancer 2009;115:1914-23.

5. Ding J, Jing X, Wang Y, et al. Thermal ablation for hepatocellular carcinoma: a large-scale analysis of longterm outcome and prognostic factors. Clin Radiol 2016;71:1270-6.

6. Ayav A, Germain A, Marchal F, et al. Radiofrequency ablation of unresectable liver tumors: factors associated with incomplete ablation or local recurrence. Am J Surg 2010;200:435-9.

7. Livraghi T, Goldberg SN, Lazzaroni S, et al. Hepatocellular carcinoma: radio-frequency ablation of medium and large lesions. Radiology 2000;214:761-8.

8. Chen $M H$, Yang $W$, Yan K, et al. Large liver tumors: protocol for radiofrequency ablation and its clinical application in 110 patients--mathematic model, overlapping mode, and electrode placement process. Radiology 2004;232:260-71.

9. Chen MH, Wei Y, Yan K, et al. Treatment strategy to optimize radiofrequency ablation for liver malignancies. J Vasc Interv Radiol 2006;17:671-83.

10. An C, Li X, Zhang M, et al. 3D visualization ablation 


\section{Page 10 of 10}

planning system assisted microwave ablation for hepatocellular carcinoma (Diameter $>3$ ): a precise clinical application. BMC Cancer 2020;20:44.

11. Li K, Su Z, Xu E, et al. Computer-Assisted Hepatocellular Carcinoma Ablation Planning Based on 3-D Ultrasound Imaging. Ultrasound Med Biol 2016;42:1951-7.

12. Zhai $\mathrm{W}, \mathrm{Xu} \mathrm{J}$, Zhao Y, et al. Preoperative Surgery Planning for Percutaneous Hepatic Microwave Ablation. In: Metaxas D, Axel L, Fichtinger G, et al. editors. Medical Image Computing and Computer-Assisted Intervention - MICCAI 2008. MICCAI 2008. Lecture Notes in Computer Science, vol 5242. Springer, Berlin, Heidelberg, 2008:569-77.

13. Floridi C, Radaelli A, Pesapane F, et al. Clinical impact of cone beam computed tomography on iterative treatment planning during ultrasound-guided percutaneous ablation of liver malignancies. Med Oncol 2017;34:113.

14. Ambrogio S, Baêsso RM, Gomis A, et al. A Polyvinyl Alcohol-Based Thermochromic Material for Ultrasound Therapy Phantoms. Ultrasound Med Biol 2020;46:3135-44.

15. Moritz AK, Winter K, Köhler C, et al. Evaluation of the accuracy of volume navigation of sonography and computed tomography using a phantom. Tierarztl Prax Ausg K Kleintiere Heimtiere 2019;47:322-33.

16. Lv S, Long Y, Su Z, et al. Investigating the Accuracy of Ultrasound-Ultrasound Fusion Imaging for Evaluating the Ablation Effect via Special Phantom-Simulated Liver Tumors. Ultrasound Med Biol 2019;45:3067-74.

17. Lafon C, Zderic V, Noble ML, et al. Gel phantom for use

Cite this article as: Zhou Y, Zhao L, Zhong X, Ding J, Zhou H, Wang F, Jing X. A thermochromic tissue-mimicking phantom model for verification of ablation plans in thermal ablation. Ann Transl Med 2021;9(4):354. doi: 10.21037/atm-21-523

\section{Zhou et al. A thermochromic tissue-mimicking phantom model}

in high-intensity focused ultrasound dosimetry. Ultrasound Med Biol 2005;31:1383-9.

18. Negussie AH, Partanen A, Mikhail AS, et al. Thermochromic tissue-mimicking phantom for optimisation of thermal tumour ablation. Int J Hyperthermia 2016;32:239-43.

19. TLCHallcrest. Permanent change thermochromic ink. Data sheet Available online: https://www. hallcrest.com/DesktopModules/Bring2mind/ DMX/Download.aspx?Command=Core Download \&EntryId=215\&language $=$ enUS\&PortalId=0\&TabId\%C2\%BC163

20. Bu-Lin Z, Bing H, Sheng-Li K, et al. A polyacrylamide gel phantom for radiofrequency ablation. Int J Hyperthermia 2008;24:568-76.

21. Butterworth I, Barrie J, Zeqiri B, et al. Exploiting thermochromic materials for the rapid quality assurance of physiotherapy ultrasound treatment heads. Ultrasound Med Biol 2012;38:767-76.

22. Dabbagh A, Abdullah BJ, Abu Kasim NH, et al. Reusable heat-sensitive phantom for precise estimation of thermal profile in hyperthermia application. Int J Hyperthermia 2014;30:66-74.

23. Singh S, Repaka R. Numerical study to establish relationship between coagulation volume and target tip temperature during temperature-controlled radiofrequency ablation. Electromagn Biol Med 2018;37:13-22.

(English Language Editor: J. Chapnick) 